International Journal of Artificial Intelligence \& Applications (IJAIA), Vol.2, No.1, January 2011

\title{
An Integrated Architecture for Enhanced Structuring of Mobile Market Place
}

\author{
Amer Ali Sallam ${ }^{1}$ and Siba K. Udgata ${ }^{2}$ \\ Dept. of Computer \&Information Sciences, A.I Lab, University of Hyderabad, India \\ amer.sallam@gmail.com ${ }^{1}$ \\ udgatacs@uohyd.ernet.in ${ }^{2}$
}

\begin{abstract}
This paper aims at presenting an integrated architecture for mobile marketplace for efficient transaction and marketing using mobile devices and related infrastructure. This architecture deals with different components of mobile marketplace and it considering a new way of integrating different functionalities of mobile market place. .
\end{abstract}

KEYWORDS: Mobile Marketplace, Buyer Agent, Supplier Agent, Search Engine, Mobile Access Mode, Payment Gateway, Security Gateway, Mobile Digital Forensic, Directory of Services

\section{INTRODUCTION}

This paper aims at analyzing and developing a general integrated architecture that supports Mobile Marketplace (MMP) rather than treating various technical aspects in isolation. MMP services are presently under transition with a record of many "tried and failed" solutions, and a promising future but uncertain possibilities with potential new technology innovation. We review the available literature on MMP, analyze the various factors that have influence on improving the quality of publishing and advertising, increasing security, enhancing the delivering processes along with payment transaction, reducing search costs and locational efficiencies in MMP. The MMP components can be categorized according to these factors. In the recent years, MMP and mobile agent technology have attracted considerable attention, and a significant body of literature has been published. A MMP is a computer program that acts on behalf of a user and moves through a network of heterogeneous machines. Its main functions [1] are: matching the requirement of buyers with available goods or services, then facilitating the exchange of information, and payments associated with market transactions. It also an institutional infrastructure, such as legal standardization and regulatory frameworks.

MMP on wireless Internet is growing rapidly. It combines Internet technology and wireless communications and in theory it can involve processes like gathering, selecting, synthesizing, and distributing of information at any time, and anywhere without any geographical constraint.

MMP is considered under the definition of Mobile Commerce (MC). MC is about delivering the right information to the right place at the right time. And it is characterized by some unique attributes that equip it with certain advantages against traditional e-commerce. These attributes can be summarized as follow:

- Ubiquity and Accessibility: Ubiquity is the main feature of MC. This involves the delivery of services in the physical world and allows the mobile user to avail these services and carry out transactions in real time in anywhere. This feature can be useful in many situations, e.g. to access any interested information or conducting transactions while standing in a supermarket or while on the move through different access mode of

DOI : 10.5121/ijaia.2011.2102 
International Journal of Artificial Intelligence \& Applications (IJAIA), Vol.2, No.1, January 2011

mobile devices whenever such a need arises. The immediacy of transaction helps to capture consumers at the moment of intention so that sales are not lost in the hiatus between the point of intention and that of actual purchase.

- Localization: Positioning technologies, such as the Global Positioning System (GPS), help with great success of providing information about mobile user's physical location at a particular moment, that can attract many service providers in particular time to offer good and services- those services include information about price and location to the item of interest, nearest hotels, restaurants, travel information, etc. - to the user based on his current location. Thus, location-based services add significant value to MC while EC cannot offer such services.

- Personalization: It seems clearly that Mobile Devices are configured for a single user where PC is often shared across resources and multiple users. This makes it possible to adjust a mobile device to the user's needs and interest via MC applications can be personalized to obtain information or provide services in ways appropriate to a specific user rather than to search for that among huge number of information that are available on the internet.

- Convenience: the features of mobile devices and their ubiquity and accessibility make them preferable tool for performing personal tasks. Thus, users are using their mobile devices more often than PCs and that considered as important clue to extend the way of interacting with people from e-commerce to more attractive way of conducting business like m-commerce. Moreover, MC extends the current traditional sales channel of EC into the more immediate and personalized mobile environment.

- Dissemination: MC offers an efficient means to targeting large number of customers by disseminate data to all mobile users within a specific geographical region.

The introduction to MMP should bring many benefits to consumers, service providers, and telecommunication operators. From the customer point of view, customers can access ondemand, at the point of purchase and obtaining best prices in the MMP. This can happen via mobile application. Although MMP provides a technology to increase merchants' sales, it enhances management efficiency for service providers. As companies exchange product information with customers in time according to their personalized preference. And that can be done via a web page promotion or a mobile alert to increase their willingness to buy a product. Also there is some portion going to telecommunication operators. The more the MMP's services are used by customers through mobile the more revenue can be achieved by telecommunication operator. In addition, the operator can also achieve revenue via fees charged to service providers for each MMP transaction. However, there is still quite a lot of issues that have to be addressed and revolved for different players in order to have adequate MMP.

\section{ARCHITECTURES OF MMP}

As stated above, the intention was to analyze and develop a general integrated architecture which can support all the aspects of MMP rather than treating various technical aspects in isolation. The invented architecture in Figure 1 illustrates the main components of MMP as layers, whereas figure 2 shows the interaction between them. The layers of MMP include wireless network, contingency theory, mobile access modes, mobile digital forensic, registration authority, security gateway, directory services, search engine, broker, auction, game theory, payment gateway, delivery agent and listening for event has been explained in coming section.

\begin{tabular}{|c|c|c|}
\hline \multicolumn{3}{|c|}{ Listening Event and Notification } \\
\hline \multicolumn{3}{|c|}{ Delivery Agent } \\
\hline Notification for Service & Transport for Goods & Delivery Time \\
\hline \multicolumn{2}{|c|}{ Store Service } \\
\hline Portal Provider & Payment Gateway \\
\hline
\end{tabular}


International Journal of Artificial Intelligence \& Applications (IJAIA), Vol.2, No.1, January 2011

\begin{tabular}{|c|c|c|c|c|c|}
\hline \multicolumn{3}{|c|}{ Payment Service Provider } & \multicolumn{3}{|c|}{ Charging Approaches } \\
\hline \multicolumn{6}{|c|}{ Security Gateway } \\
\hline Confidentialit & \multicolumn{2}{|c|}{ Authentication } & Inte & & Non-repudiation \\
\hline \multicolumn{3}{|c|}{ Broker } & \multicolumn{2}{|c|}{ Auction } & \\
\hline Negotiation & Matching & Filtering & $\begin{array}{c}\text { Auction } \\
\text { Type }\end{array}$ & Information Available & Game Theory \\
\hline \multicolumn{6}{|c|}{ Search Engine } \\
\hline \multicolumn{2}{|c|}{ Products } & \multicolumn{2}{|c|}{ Services } & \multicolumn{2}{|c|}{ Catalogs } \\
\hline \multicolumn{6}{|c|}{ Directory of Services } \\
\hline Publishing & \multicolumn{2}{|l|}{ Advertising } & LBS & Map & Profile-Based \\
\hline \multicolumn{6}{|c|}{ Mobile Digital Forensic } \\
\hline \multicolumn{3}{|c|}{ Buyer Agent } & \multicolumn{3}{|c|}{ Supplier Agent } \\
\hline \multicolumn{6}{|c|}{ Mobile Access Mode } \\
\hline Web Mode & Asyn & s Mode & Notificat & Mode & ice Mode \\
\hline \multicolumn{6}{|c|}{ Contingency Theory } \\
\hline Social / C & & ercial & Technological & Regulation / St & dardization \\
\hline \multicolumn{6}{|c|}{ Wireless Infrastructure } \\
\hline Cellular & & ANs & Public & & lite Service \\
\hline
\end{tabular}

Figure1: Layers structure of MMP

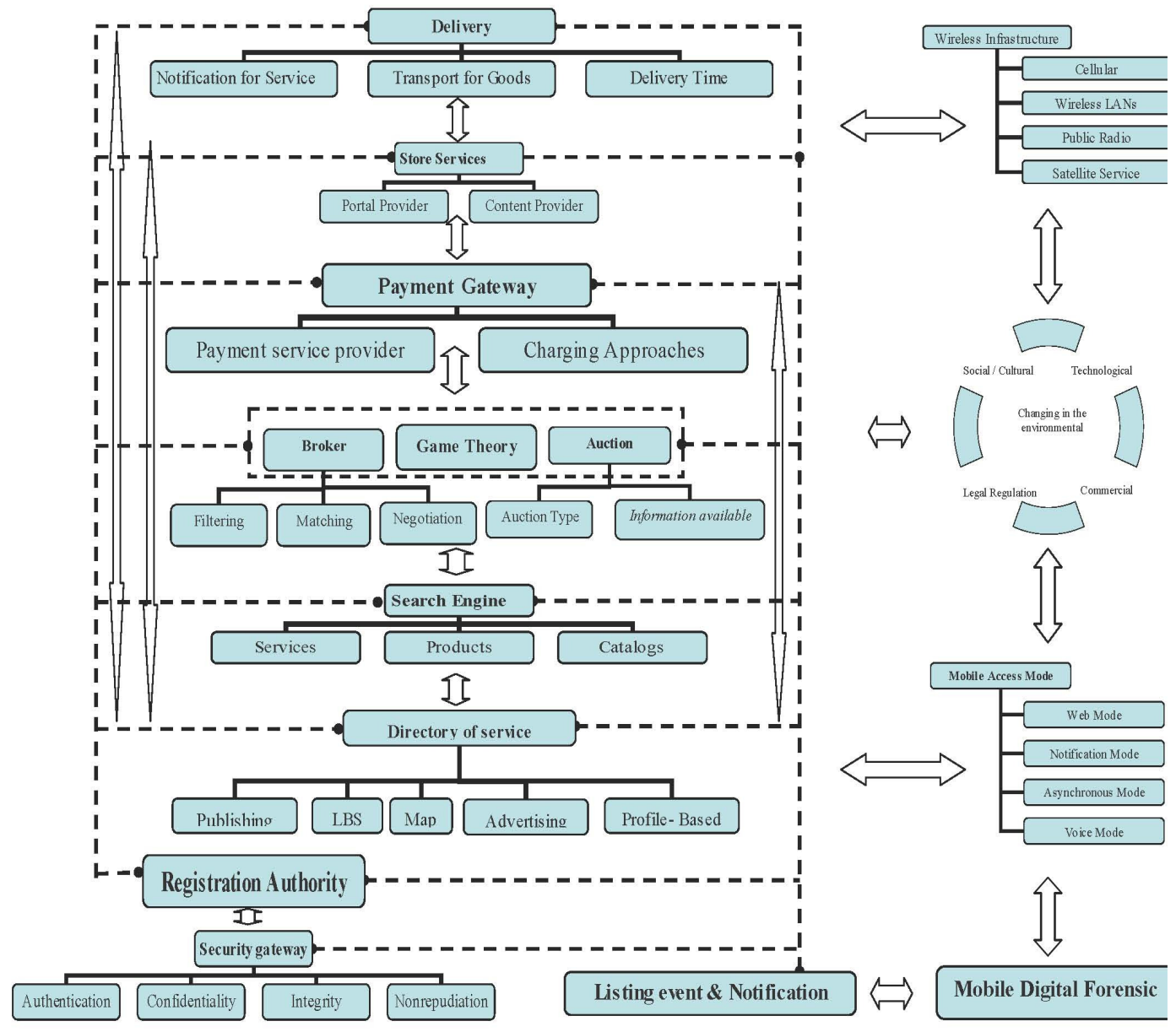

Figure2: The architecture of Mobile market place and the interaction between their components 
International Journal of Artificial Intelligence \& Applications (IJAIA), Vol.2, No.1, January 2011

\subsection{Wireless Network}

Generally, the network infrastructure for wireless mobile combines different wireless networks, involving point-to-point wireless bridges, wireless LAN, private and public radio, satellite services, wireless local area networks, multidirectional wireless cellular systems). As well, there are many types of wireless mobile devices (e.g. portable computers, smarts cellular phone, Bluetooth communication device, PDAs, Internet terminals, etc.). These devices have the ability of conducting the desired transaction and preferences of MMP. At the meanwhile, wireless systems are achieving higher data rates to support Internet and other data-related applications. Through these networks, consumers and merchants are connected and they are able to manage and accomplish the transaction that is preferable by them. The wireless mobile communication devices associated with the potential consumer can receive communications from merchants, either directly or via an intermediate system, and can transmit a reply back to the merchant indicating a proposed offer so that a merchant can revise or improve the offer.

However, Mobile wireless communication devices do not normally always communicate with the network infrastrucure, i.e., they are unreachable for many reasons according to the consumer desire, viz, when the user is in a meeting, sleeping, or even to reduce power consumption of the mobile or there is no network coverage. In addition, Wireless communications are still facing challenges in transfer capacity when it is compared to wired networks. This is caused by the fact that the modulation used and channel allocation schemes designed for voice traffic have rather modest upper bounds. Further, the wireless communications are much more error prone than the wired communications and require much redundancy in the channel coding of the payload. Although the redundancy in the channel coding that makes correcting bit errors in a wide range possible at the receiving end, in wireless retransmission of the data is required more often than in the wired networks. .

\subsection{Contingency Theory}

As a matter of fact, MMP as any trade has been influenced by some external factors that have been gathered under the theory of contingency. This theory has been used for classifying mobile payment research and to capture the environmental factors which are characteristic to the mobile payment services markets. Furthermore, it has emphasized the importance of environmental influences, especially technology, on the management of organizations. In addition to technology, other typical contingency factors include cultural, social and economic factors. In the context of mobile payment service markets, because financial services and telecommunication are among the most regulated industries, it is natural to include regulation, jurisdiction and standardization factors, and the use of standards is characteristic to telecommunication [2]. Contingency theory is described as a midrange theory which falls between two extreme views [3][4]. According to one extreme, it is possible to find universally true theories, whereas the other one claims that each unit of analysis is unique and has to be analyzed based on situational factors. Further, Contingency theory postulates that environmental factors are only important but also that the impacts of environmental factors are systematic, rather than entirely situational. The other helpful feature of contingency theory can be manifested via the "environment - strategy - performance" link. The theory claims that the environment, such as the amount and type of regulation, impacts the structure of the organization, for example, influencing which entities have incentives to become mobile payment service providers. Another example is that enhanced technology makes it possible to provide enhanced services which in turn increase interest toward the services. These contingency factors have significant impacts on the mobile payment services market but they are outside of the influence and control of the market. 


\section{Changing Commercial Environment}

At the outset, changes in the commercial environment embrace the development of the Internet and mobile networks into commercial channels, as well as increasing automation and self-service orientation of payment services [5]. Changes of the commercial environment may operate on the development of the new or the improved mobile payment services [6]. Other aspects of this factor include the structure and development of financial and telecommunication infrastructures and markets within the studied environments. The development of mobile payment services might be supported or inhibited by the structures of financial services markets within various countries may support or inhibit.

\section{Changing Social \& Cultural Environment}

It is widely argued that a specific part of the overall culture and lifestyle of the society is that of the payment culture. Social and culture environment plays an essential role in affecting people's consumption habits, buying behavior, and thus their needs for new payment services. Changes in these environments [6] can deal with varieties of needs and thus affecting the supply and demand of new payment services.

\section{Changing Legal Standardization \& Regulatory Environment}

Legislation and standardization of mobile payments according to current researches provide an informative description about the difficulties and the problems surrounding such topics. Yet, no significant solutions can solve the issues of legislation and standardization. Cross-border mobile transactions can be complex due to a complicated web of law and regulations. These contingency items may trigger needs for new or enhanced payment services and drive or hinder the development of mobile payments [6] [7].

\section{Changing technological environment}

Technological environment consists of wireless and other related technologies which are used to develop and produce mobile payment services. Some of these technologies such as mobile network technology or transaction protocols are regarded among the technologies which develop slowly. Some other technologies have very short development cycles, such as mobile handsets and their components. Constant development of technologies facilitates more reliable, user friendly, versatile, and functionally rich mobile payment services.

\subsection{Mobile Access Mode}

The modes of mobile access such as asynchronous, online web, notification, and voice mode apparently do not have to be used in isolation but can be combined within an application design [8].

\section{Notification Mode}

The notification includes other appealing services such as instant messages on Internet, SMS/MMS messages on a GSM/GPRS cellular network. In order to be effective, this mode enables e-commerce features such as real-time message alerts on a very small portable device and it requires the mobile network to be "always-on." A message gateway is installed to offer different messaging communication stacks, and perform message queue management and notification event management.

\section{Asynchronous Mode}

The asynchronous mode enables users to download content to MDs and operate the content offline without network requirement, irrespective whether they have occasional network 
connectivity or are only connected to a PC for synchronization in the office or base location. Moreover, it could greatly enhance the user experience for m-commerce especially when the wireless network bandwidth is slow or the quality of the wireless signal is poor. This mode is very popular in current generation of PDAs. The MD under the consideration on this mode can synchronize with the sync gateway based on IP connectivity to a sync server, or if that gateway is on a PC, using a serial cable, USB, Bluetooth, or infrared connection. The sync gateway can connect the device to a personal productivity data, i.e., Calendar, to-do lists, memos, e-mails, documents, web content, database and application messaging. Regarding Web content, the sync gateway allows the MD to synchronize personalized Web content. The personalized selection is determined using the synchronous mode, usually through a PC. The user selection can be associated with a profile in the Directory service.

\section{Online Web Mode}

Mobile users can access MMP applications via a web browser on MDs like the PC users. But different MDs may adopt different display features and communication protocols. Gateway in the MMP architecture should be deployed to support various pervasive devices. A wellknown example of this topology is called a WAP-enabled device with a cellular network and a gateway that includes the WAP gateway software. The WAP gateway is responsible for transforming a WAP request into an HTTP request to the web application. The most common configuration of the gateway might be through cellular network provider and connected to the internet. This gateway configuration is important for an ISP to provide a set of public services to subscribers. It is also possible for this gateway to be configured by the mobile network provides via direct connection to that gateway and does not involve the public Internet. This gateway configuration is relevant for a solution to a service intended only for an enterprise group requiring mobile access to $\mathrm{m}$-commerce services.

\section{Voice Mode}

With the limited keypad space and screen size of MDs, voice technology promises improved input and output functionality for m-commerce. The voice mode of interaction with an application requires speech recognition and text-to-speech synthesis (TTS) technology. However, unlike personal computers, the current generation of MDs does not have the processing power to perform these transforms.

\subsection{Mobile digital forensic}

Digital forensics concerns with investigation of cyber crimes and establishing the identity of intruders and gathering evidence of malicious activity. If this unit has enough evidence [9] to prove that somebody did try to attack the security transaction of MMP, then it will further investigate for presentation to courts of law. Digital forensic emphasizes that the rules and the protocols are shared and must be understood and followed by everyone relying on it, can be guaranteed to attain the commitments of participants, otherwise will receive appropriate penalty. It is necessary for a market institution to be equipped with capabilities that will prevent the occurrence of cyber crimes from happening, or if they do happen, they will keep or log the evidence, and report the crimes.

\subsection{Registration Authority}

In the MMP, Registration Authority (RA) deals with registration of buyers, suppliers, service providers, etc. The participants send their request for a registration which brings along the request, their certificate, and other necessary information to registration authority. RA performs verification of participants' certificates through the Certificate. Certificate is a trusted 
third party to provide validity of the secrets key which are used for authentication. Those digital certificates used to proof the identity and authentication of each MMP units.

\subsection{Security Gateway}

Security gateway becomes the entry and exit points with the process of migrating or transmitting in the MMP components. However, the security is one of the critical issues for successful adoption of payment transaction and it needs to be confidential .Confident transaction in the MMP means that both business and individual consumers have to be sure that the risk of fraud is minimized or there is no risk at all, providing higher level of security to protect mobile payment transactions, taking into account to prevent any trial of fraud payment transaction from stolen devices. Therefore, to make that security system trustable, it should involve some criteria as follows [10] [11]:

Confidentiality and identification: this means that electronic messages which are sent with unique identification information for verification must not be visible to eavesdroppers and underlying on the mobile network security.

Authentication: where service provider should authenticate the transaction from users via an identification process or cryptographic mechanism. In other words, MMP units must identify each other's identity, reliance on the personal nature of a MD and reliance on authentication by mobile network operators.

Integrity and Non-repudiation: which means MMP units must know when the data they send have been obtained and it must be possible to prove that a transaction has taken place.

Secure Performance, the service provider has the responsibility to ensure that the requested transaction is performed under a secure environment and ensure a safe protocol for payment transfer.

\subsection{Directory service}

The Directory service responsible for publishing and advertising goods and services offered by the existing brokers and provide channels to communicate with other MMP units which assist to obtain useful information about location and maps, which can help to point the exact position of the product in the market, and gives information about location and cities roads. Also user or merchant based profile could be useful to provide information about user requirement, merchant offers or product specification. All these components gather information to supply for directory service.

\section{Advertising}

The technology that enables communication between MMPs via present typical message contains product or service information either huge or small is called advertising. This technology consists of different forms of advertisement. According to the personal needs or integration, it might be advertising one or list of different broker, auction, service provider, product or service among different MMPs. In other words, it provides service to suppliers who could not find any matching buyer registered under the same MMP for a product or service that is being offered. In addition, through advertising MMP can attract more customers and dramatically improve sales and business makes more profit.

\section{User Profile/ Merchant}

User Profile means a database in which personal data of users or merchants are stored. These data can include a user name, address, prefrences, IP address, merchant location or equivalent. 
International Journal of Artificial Intelligence \& Applications (IJAIA), Vol.2, No.1, January 2011

This data are used to establish a connection to the users and to send user's interests to the merchants or to figure out whether one of the user is close to registered merchant. User profiles contain the user's interest and what he/she is searching for. In addition, user profile can look for or concern related products in the product database which are useful to be used with the current selected product by any user. However, it may try to ignore to send any advertisement for the product already taken place by user, unless the user has sent another request for the same product. It helps prevent receiving undesirable or disturbing advertising. What is more is that it can sort and categorize users according to their profiles (e.g. age, profession, product of interest, selected product, etc) which may invent new improvement in MMP, i.e, according to selected product, advertisement units could help to provide suitable advertising for the related products timely or in future which may enhance the ability of business interaction between merchant and customer from one hand, and obtain information and new features about available and related products (e.g. accessories, terminal, etc) with selected product on the other.

\section{Maps}

By maps, it is meant the map database stores which draw information of cities and the exact position of the product, shopping malls within stores. This information is required to produce directions to a given product.

\section{Location-based services}

Mobile network Location-Based Services (LBS) provide a service to target supplier or buyer based on their physical [12] location and presents information about that to the concerned different units in MMP to improve their services according to what is needed. Nowadays most of smarts phones and mobile devices support the GPS system which is very helpful to gather information about locations. Here, it is possible that subscribers could soon be placed with near pinpoint accuracy. In this regards, such service still not preferred by some consumers as it does reveal their personal privacy. However, location information has been the monopoly of the carriers and network operators themselves. In the future, this may not be the case. For example, Bluetooth or WiFi can contribute to location determination, in that a network fixed devices may constantly communicate with MDs over a Bluetooth network or wireless LAN.

\subsection{Search engine}

No doubt that Internet and the wireless supply an actual MMP all over the world are without location and time restriction. Search engine is very helpful unit in MMP. It provides the necessary information to various units of MMP [13][14] with respect to their request. Usually, mobiles MMP make available on the Internet electronic catalogs that support lists of products (such as book, flowers, etc.) or services (e.g. news, ticketing services, financial services, etc.) price information and commercial transactions. Depending on that, search engine will return a list of products or filtered services which meet the requirements specified by the buyer. According to the huge number of agencies i.e., broker, auction, delivery, payment, supplier, buyer, etc and overloaded information interacted and presented between them, search engine is considered the backbone of MMP and important unit.

\subsection{Broker, Auction and Game theory}

This unit contains different components available as separated or combined. Whatever the forms of their availability were, it has to work with integration.

\section{Broker Agent}

Broker agent is considered a party that mediates buyers and sellers in a MMP. However, it developed to summarize the results and present it to users via searching through MMP for price 
and characteristics of the products or service. Therefore, it performs different tasks such as matching buyers and sellers [15], filtering the offer information about goods or services' specification and prices [16], register with a certain auction type as the price negotiation mechanism is to match suppliers with buyers. Likewise, it helps buyer agent to search for a desired goods or services with specific criteria, then retrieving specific information (e.g., price) to help him determine what to buy, and to decide whom to buy from. The main idea of broker is to obtain multiple searches accomplished by agents working in parallel along with information sources together in one place.

\section{Auction}

The term 'auction' means the process with an explicit set of rules of buying and selling goods or services, by offering them up for bid, to determining resource allocation and prices, taking bids, and then selling the item to the winning bidder [15] [17]. In economic theory, an auction may refer to any mechanism or set of trading rules for exchange. Auctions have been recognized as an excellent trading mechanism to allocate resources, viz, goods, services, etc. to individuals and firms [18]. In addition, Auctions can be distinguished in a number of participants [16]: The first is in supplying (or reverse) auction, where m-sellers offer goods that a buyer requests. The second is in demanding auction, n-buyers bid for goods being sold; the third is in doubling auction $\mathrm{n}$ buyers bid to buy goods from $\mathrm{m}$-sellers.

\section{Types of auction}

There are many types of auctions, the most well-known of which will be mentioned briefly as follows:

English auction which is identified as an open ascending price auction. This type of auction is arguably the most common form of auction in use today.

Dutch auction identified as an open descending price auction, while the auctioneer begins with a high price and lowered until some participant is willing to accept the auctioneer's price.

Sealed first-price auction known as a first-price sealed-bid auction. In this type, all bidders simultaneously submit sealed bids so that no bidder knows the bid of any other participant. The highest bidder pays the price they submitted.

Vickrey auction well-known as a sealed-bid-second-price auction. This is identical to the sealed first-price auction except that the winning bidder pays the second highest bid rather than their own.

\section{Game Theory:}

Game theory can improve the system of negotiation filtering the competition result performance of goods and services in MMP. The scenario of this game is, firstly, each player estimates the number of competing nodes (i.e. game state). Secondly, based on this state, each player tunes its equilibrium strategy by changing its local contention parameters. Finally, the game is repeated until to get the optimal results.

\subsection{Payment Gateway}

The Payment Gateway means a trust third party that is responsible for processing the payment transaction from initiation till completion of payment with authorization. Mobile payment has two tasks: (1) inside m-commerce and (2) outside m-commerce. The first task could occur when the scenario of m-commerce reflects the billing and payment for direct transaction-dependent in m-commerce. This includes mobile value-added services and the purchase of non-digital goods or services via the mobile channel. Typical examples of this are 
news, financial information, and entertainment. There are two well-known offer models for mobile value-added services, one offer by the mobile network operator (MNO), and the another one direct offer by a mobile content provider (MCP).The MNO produces mobile content or services itself or buys them from a MCP acting as a supplier and thus offering a single face to the customer for network and all other services. M-payments, in contrast to $\mathrm{m}$ commerce functionality, compete directly against other payment systems such as e-payment, credit or debit card, or cash.

\section{Current charging approaches for payment gateway}

There are three essential charging approaches to the payment gateway:

The sponsoring approach, performs a particular task. Services are free of charge for customers because they are provided at the mobile content provider's expense.

Premium fee charging, here, customers pay a data volume fee for transmission and additionally a so-called premium fee for the value of the content or service. The MNO as payee gets the data volume fee and transfers the premium fee to the MCP after deducting a compensation for its costs. "Order last goal", would be a typical example to provide a certain number to receive a multimedia message back, including a video clip of the goal.

Fixed price charging, here, customers pay a fixed price for their usage of the service. This revenue as a whole is shared between $\mathrm{MNO}$ and $\mathrm{MCP}$ according to an agreed upon formula.

\section{Payment Service Provider}

Payment service provider provides the payment procedure to the customer as well as to the portal provider or to transport service provider. The authentication service provider, in turn, provides the verified customer identity to the mobile payment service provider.

\subsection{Delivery Agent and Store Service}

The delivery agent is considered as a trusted third party which is responsible for delivering goods and services. The transport service provider provides data transport to the customer and thereby forwards the product to the customer after getting the details of customer information contact through supplier agent.

The store service (portal), a site bringing together a variety of content and services in one area and attracts a large number of visitors, delivers the product to the transport provider and pays compensation for payment handling to the payment service provider, typically as a transaction fee.

\subsection{Listening, event log and notification agent}

This unit provides the necessary mechanism on the way, topic, time and place to record the activities related to transactions carried out by the Buyer and Supplier Agent, based on the agreed privacy policy achieved during the privacy negotiation process. To record this information to be used for mobile forensic investigations [11], log files are used.

\subsection{Buyer Agent}

Buyer agent is a mobile bearing its owner's information, certificate, privacy policy, product/service request [19]. Buyer agent's information includes a unique identification, the broker's registration, a contact address, bank account particulars and bid-related information. To enter the MMP, it has to be authenticated by the security gateway, negotiating privacy policy to 
hide its identity and protect confidential data, then, forwarded by the Directory Service to its broker bidding for the requested product/service and returning to its owner when its request has been fulfilled.

\subsection{Supplier Agent}

The mode of operation of supplier agent is similar to buyer agent. It returns to its owner when a trading partner has been found. A Supplier Agent is called a mobile agent who sends requests for its products/services to be advertised and sold. It carries its owner's information, certificate, privacy policy and information on products/services features, reserved price and time limit [19].

What a broker agent has to do is respond to Buyer Agent's requests, acknowledge the Buyer Agent of previously requested information, choose a supplier according to Buyer's specified criteria, and communicate with other brokers. If a product/service requested by a buyer is not available on its supplier's list, the broker will send a message to other brokers querying for the specified product/service.

\section{NOVELTY OF THIS ARCHITECTURE}

E-marketplace refers to the field of marketing, selling, buying and distributing different products or services over the internet but still lacks flexibility and convenience, this type of MP are limited according to convenience, diversity of accessibility, as well as in the personalization where the PC is often shared across multi-user. However, the emerging technology of MMP is promising and could lead to more user satisfaction. While MMP on wireless internet is growing exponentially, it uses two highly powerful and new technologies, viz, internet and wireless communication which involves gathering, selecting, synthesizing and distributing of information at any time and anywhere without any restriction of geographical place.

\section{CONCLUSION AND FUTURE WORKS}

On this paper we analyzed the various components of mobile market place. We demonstrated the heterogeneity in the different components. The main contribution of this paper deals with development of a general integrated architecture, by focusing on the heterogeneous nature of the components. It is intended to highlight the difference components, their relationship, importance in a mobile market place. Through this architecture we aim to improve the quality in term of publishing, advertising, increased security and efficient delivery mechanism along with Payment mechanism.

\section{REFERENCES}

[1] Ibrahim, I. K., Schwinger, W., Weippl E., Altmann J., Winiwarter W. (2001). "Agent Solutions for E-business Transactions," Proceedings of the 12th International Conference on Database and Expert Systems Applications (DEXA2001), Los Alamitos, IEEE Computer Society Press.

[2] Tomi, D., Niina, M., Jan, O., Agnieszka, Z. (2008). "Past, present and future of mobile payments research: A literature review”. Electronic Commerce Research and Applications, 7, pp. $165-181$.

[3] David, M. G., Frank, J., Moonkyu, L., Ian W. (2000).” A contingency approach to marketing high technology products," European Journal of Marketing 34 (9/10), pp. 1053-1077.

[4] Valarie, A., Z., P., "Rajan" Varadarajan, Carl P. Z. (1988)." The contingency approach: its foundations and relevancy to theory building and research in marketing," European Journal of Marketing 22 (7), pp. 37-64. 
International Journal of Artificial Intelligence \& Applications (IJAIA), Vol.2, No.1, January 2011

[5] Hampe, Paula, M.C. S., Paul A. S.( 2000). "Mobile electronic commerce: reintermediation in the payment system, in," Proceedings of the 13th Bled eCommerce Conference, Bled, Slovenia, June 19-21.

[6] Jayawardhena, C., Foley P. ( 2000). "Changes in the banking sector - the case of Internet banking in the UK," Internet Research 10 (1), pp. 19-31.

[7] Rawson, S., (2002). "E-commerce mobile transactions: mobility and liability: the hazards of handhelds," Computer Law \& Security Report 18 (3), pp. 164-172.

[8] S., Song (2006). "Mobile Commerce and Wireless E-Business Applications," IBM China Research Laboratory, pp. 335-359.

[9] Giannakis, A., Leon S., Stefanos G., Parampalli U. (2006). "Network Forensics protocol embedding Privacy Enhancing Technologies," Proceedings of the ISCIT International Symposium on Communications and Information Technologies, A. Taguchi et al. ed., , Bangkok, Thailand.

[10] Norleyza, J., Mazliza, O., Rodziah, L. (2002). "Secure Agent-based Marketplace Model for Resource and Supplier Brokering," Proceedings of the 2nd Asian International Mobile Computing Conference (AMOC), May 14-17, Langkawi Island, Malaysia.

[11] Norleyza, J., Noor, Y., Yazrina, Y., Ahmed P., Mazliza O. (2008). "Secure and auditable agentbased e-marketplace framework for mobile users," Computer Standards \& Interfaces, 30, pp. 237-252.

[12] Dimitrios, M., Emiris, Charis, A., Marentakis, Panayiotis, P., Laimos (2007). "Towards An Integrated Lbs-Enabled, Mobile Auctions Marketplace For Logistics Services," 18th Annual IEEE International Symposium on Personal, Indoor and Mobile Radio Communications (PIMRC'07).

[13] Ibach, P., Tamm, G., Horbank M. ( 2005). "Dynamic Value Webs in Mobile Environments Using Adaptive Location-Based Services," 38th Hawaii International Conference on System Sciences, Hilton Waikoloa Village Island of Hawaii, Big Island.

[14] Tilson, D., Lyytinen K., Baxter R. (2004)."A Framework for selecting a Location Based Service (LBS) Strategy and Service Portfolio," 37th Hawaii International Conference on System Sciences, Big Island.

[15] Kannan, P.K., Chang, A.M., Whinston, A.B. (2001). "Wireless Commerce: Marketing Issues and Possibilities," 34th Hawaii International Conference on System Sciences, Maui, Hawaii.

[16] Jailani, N., Abbas, M.F., Othman, M., Zakaria, M.S. (2006). "Bidding agent strategies in a Dutch auction marketplace," Proceedings of the 4th Asian International Mobile Computing Conference, Kolkata, India, pp. 287-294.

[17] McAfee, R., McMillan, J. (1987). Auctions and bidding, Journal of Economic Literature, 25, pp. 699-738.

[18] Klemperer, P. (1999). "Auction Theory: A Guide to the Literature, Journal of Economic Survey," 13, pp. 227-278.

[19] Timon, C., Du, Eldon, Y., Lib, Eric, W. (2005). "Mobile agents for a brokering service in the electronic marketplace,” Decision Support Systems, 39, pp. 371- 383.

\section{Authors}

Amer A. Sallam is a Ph.D. scholar in computer science at the University of Hyderabad, in the Research Scholar Lab under the supervision of Dr. Siba K. Udgata. His research activities focus on the design and implementation of adequate programming abstractions for emerging distributed architectures, such as mobile marketplace with a focus on recommendation and publishing systems.

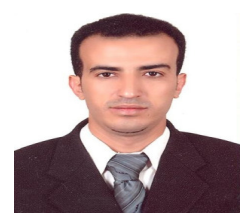


International Journal of Artificial Intelligence \& Applications (IJAIA), Vol.2, No.1, January 2011

Siba K. Udgata is an associate professor in computer and information sciences, university of Hyderabad. His main research activities focus on mobile computing, wireless sensor network and Ad hoc network. He was an united nations fellow and currently working on research projects for development of WSN application and resource allocation schemes for cognitive radio network.

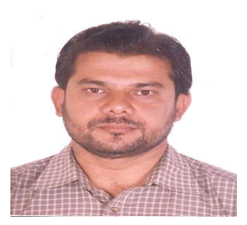

